Pol J Med Phys Eng 2012;18(2):49-58.

PL ISSN 1425-4689

doi: 10.2478/v10013-012-0007-x

website: http://versita.com/pjmpe

${ }^{1}$ Hiba Baha Eldin Sayed Omer

\title{
Intensity modulated radiotherapy using Monte Carlo for routine postmastectomy radiotherapy
}

\author{
${ }^{1}$ College of Medicine, University of Dammam, Saudi Arabia \\ e-mail: hibaha@yahoo.com
}

\begin{abstract}
Radiotherapy given after mastectomy (PMRT) will reduce the risk of local recurrence by about two-thirds. Clinical and dosimetric trials were carried out using various techniques to optimize the treatments by maximizing the dose to the tumour and minimizing it to the healthy tissues at proximity. Different conventional techniques which have been studied suffer from important dose inhomogeneities due to the complex anatomy of the chest, which reduces the benefits from such treatments. Moreover, due to the heterogeneity of breast cancer, the response to therapy and a systematic approach to treatment cannot be derived and treatment regimens must be determined on a patient-by-patient basis. This is only possible if accurate and fast treatment planning systems are available. Intensity Modulated Radiotherapy (IMRT) allows delivering higher doses to the target volume and limits the doses to the surrounding tissues. The objective of this study is to test the feasibility of applying a Monte Carlo-based treatment planning system, Hyperion accurately in routine Intensity Modulated Radiotherapy (IMRT) postmastectomy. In order to use a treatment planning system for routine work it should prove to provide optimized dose delivery in a suitable time. Treatment planning for IMRT application to PMRT was performed using Hyperion. Constraints were set to deliver the prescribed dose to the target and minimize the dose to the organs at risk. Dose Volume Histograms (DVH) were used to evaluate the set up plans. Time taken to optimize the plan was measured. The target coverage was within the accepted values. Approximately $90 \%$ of the breast and $80 \%$ of the PTV received 45 Gy or above. The volume of the lung that received $40 \mathrm{~Gy}$ was less than $10 \%$ and the volume that received 20Gy (V20) was less than $25 \%$. The volume of the heart receiving 30 Gy (V30) or above was negligible. This indicates low NTCP of these organs. The time taken for optimization, showed it possible to apply Monte Carlo-based treatment-planning systems for patient-to-patient PMRT.
\end{abstract}

Key words: PMRT, IMRT, Hyperion, Postmastectomy Radiotherapy

\section{Introduction}

Even after mastectomy, the risk of local recurrence can remain unless some reliable method of investigation, such as axillary clearance, has found no evidence of nodal involvement. If nodal involvement is present, Post-Mastectomy Radiotherapy (PMRT) can produce a substantial absolute reduction in this risk of local recurrence [5]. 
Breast radiotherapy is generally estimated to constitute at least one-third of all work in radiation oncology and, as such, constitutes a large portion of work in any department. Radiotherapy of the breast is a complex and demanding task due to the heterogeneity of the target volume. Over the recent years, different conventional techniques have been established but they suffer difficulties in balancing an adequate irradiation of the internal mammary chain with the sparing of lung, heart and contralateral breast. IMRT can achieve this balance and reduce irradiation of organs at risk [7].

IMRT techniques employ inverse planning methods and biological complication models for organs at risk. It is driven by computer-optimized planning that allows modulation of beam intensity within treatment fields to obtain highly conformal dose delivery. This removes the usual reliance on flat (or uniform intensity) radiation fields and, instead, replaces that simple paradigm with a variable intensity pattern that is usually determined using a computerized optimization algorithm. Complex dose distributions are achieved using irregular fluence maps obtained through optimization processes. The irregular fluence is delivered by a superposition of several segments of beams during the step-and-shoot procedure [6, 1].

The objectives of this study are to evaluate the efficiency of Hyperion, a Monte Carlo-based treatment planning system that has been integrated from XVMC in solving the challenges in PMRT treatment planning on a patient-by-patient basis.

\section{Materials and Methods}

IMRT plans were defined in Hyperion [2]. Hyperion is an IMRT planning system based on constrained biological optimization and a gradient method to calculate the primary fluence updates. The concept of biological optimization relies on a classification of complications as ultra-parallel, serial and parallel [6, 1, 2]. A CT data set from stage II breast cancer patient who underwent radical mastectomy was chosen for this study. The treatment planning starts with the definition of critical organ structures on several slices of the CTs to ensure consistency within each structure and corresponding maximum damages measured by an effective dose. The implemented optimization algorithm tries to maximise the dose and thereby damage in the tumour volume by retaining the organ constrains, which can be changed by the user during the planning process.

The optimization process employed by Hyperion is divided into two stages. The first stage results in fluence profiles based on Pencil Beam (PB) dose calculation. In the second stage first the beamlet-based fluence profiles are converted to segments, then optimized, then the segment-doses are calculated with Monte Carlo (MC) and finally some re-optimization based on the MC-segment-doses is performed [2, 12]. 
A field arrangement consisting of six $6 \mathrm{MV}$ photon beams from an ELEKTA multileaf collimator having 80 leaves was performed at angles $0,20,60,-25,-90$ and $-132^{\circ}$ around each patient in the axial plane. The optimization setup included hard constraints for the maximum dose to the target, in order to maximize the Equivalent Uniform Dose (EUD) under the given constraint and hard constraints on the maximum EUD of the organs at risk. A Dose of 50 Gy in 25 fractions was prescribed for the planning target volume. The parallel and serial reference doses to both lungs were set to 20 and 40 Gy respectively. The maximum dose to the whole heart was set to $48 \mathrm{~Gy}$. Cost-functions were also set to the myocardium, contralateral breast, spinal cord, liver, stomach and intestine. Figure 1 shows the input window for the description of the first plan. Dose volume histograms were used for evaluation of the plan. The time taken for the optimization procedures (both pencil beam and Monte Carlo processes) was observed

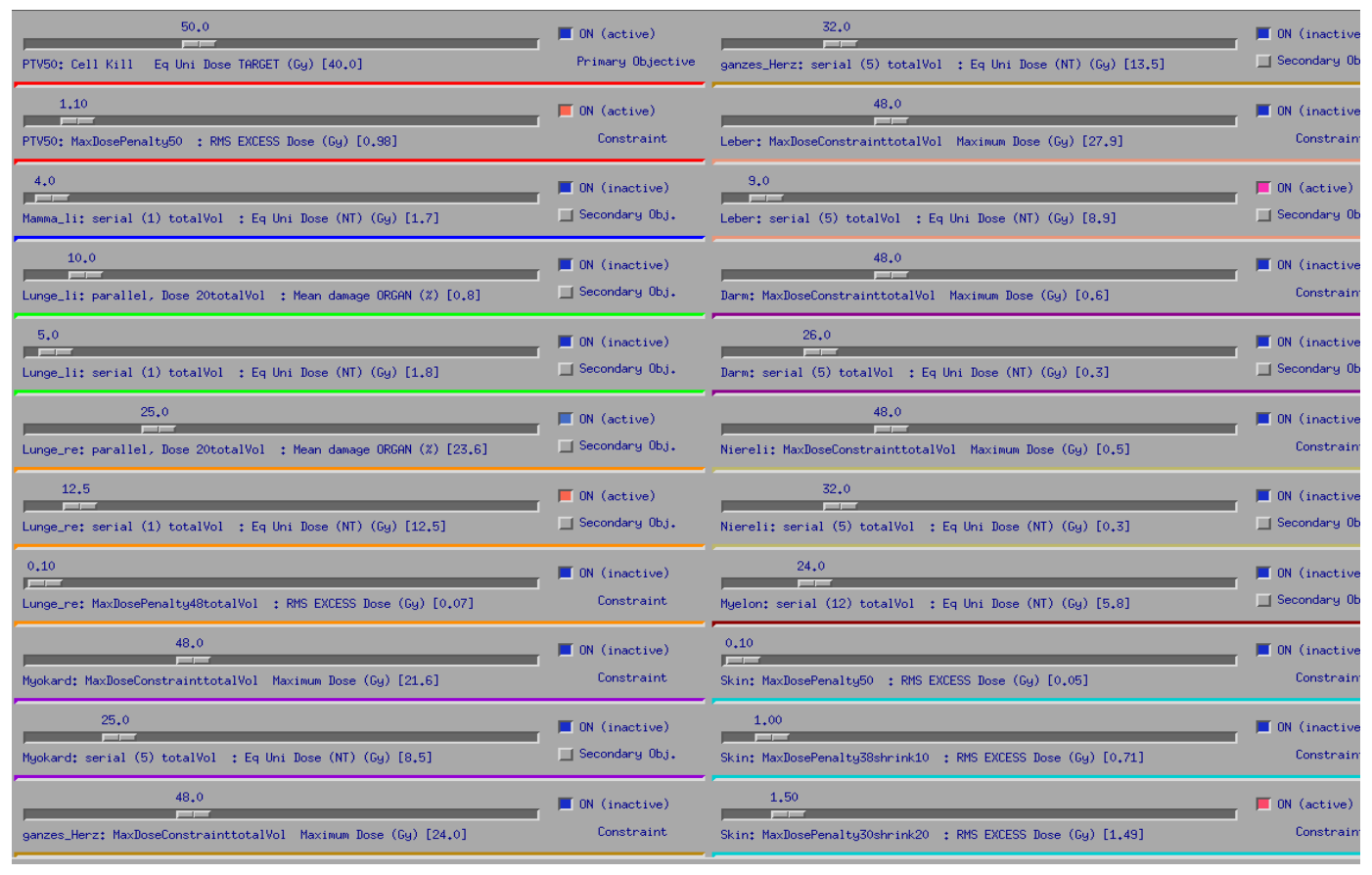

Figure1. Description of the first plan

For the second CT data sets, similar constraints and same CLN file were used and the time to perform the optimization was measured. The constraints in the right lung and heart were then changed with a significant variation in constraints values in one CT set, to measure the time required when big changes are made. This is referred to as the third plan. In a fourth plan slight variations in the PTV constraints were made to measure the time needed for planning with slight variations to adapt to account for the variability in each patient's needs. 


\section{Results}

\section{Target coverage and risks to healthy organs}

Factors such as target coverage as well as the dose received by certain volumes of the lung (V20) and heart (V30) were considered in this study as will be discussed later. The optimized results for this, second plan, CT data set, the isodose curves and DVH are shown in Figures. 2-4, respectively.
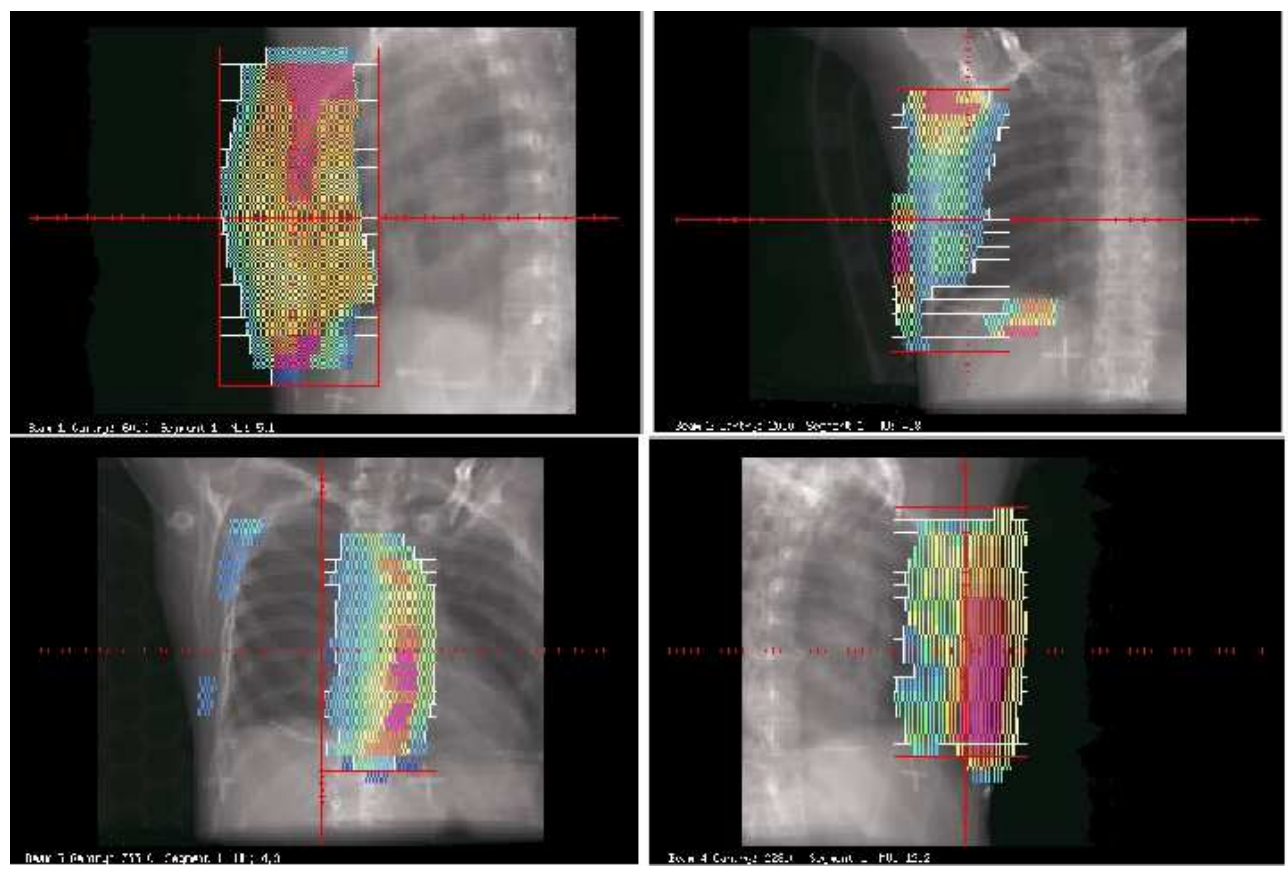

Figure 2. The segment of the different beams of the second plan as calculated by Hyperion

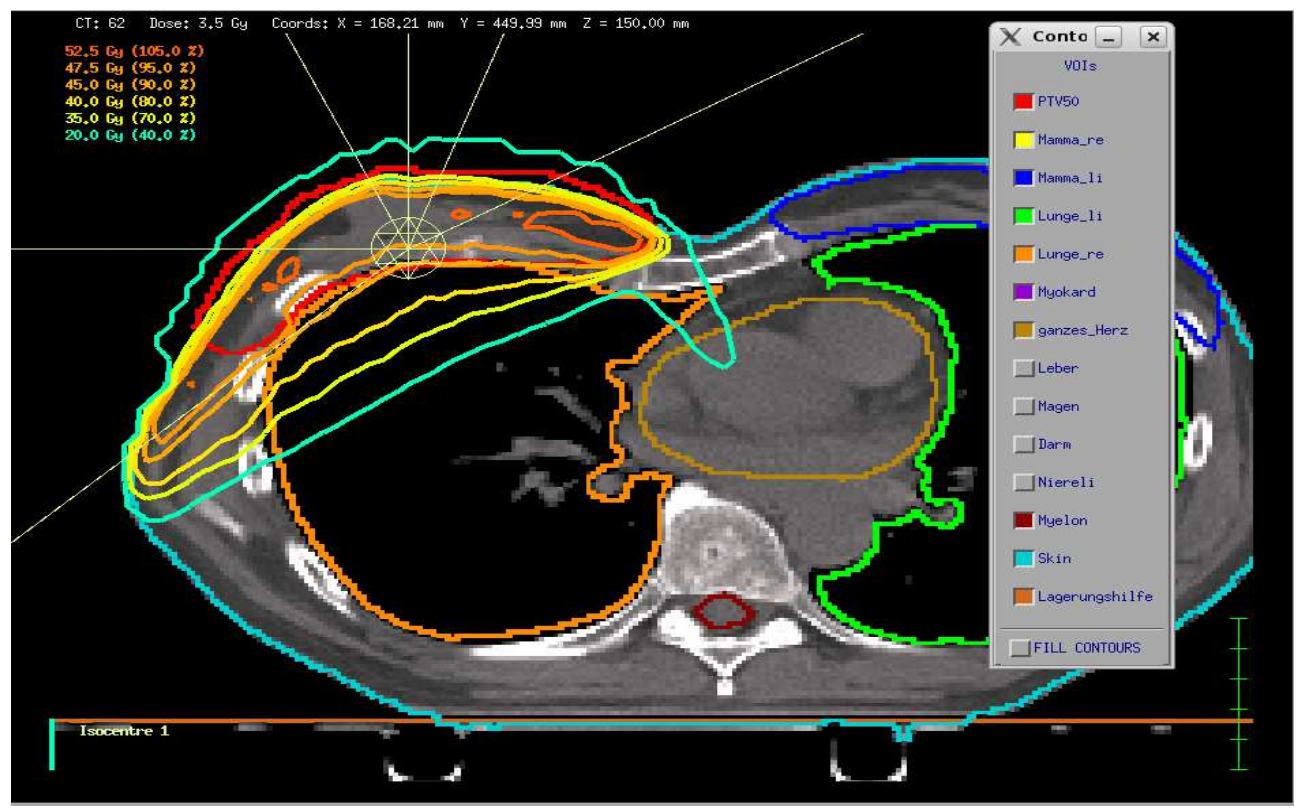

Figure 3. Isodose curve of the second plan 


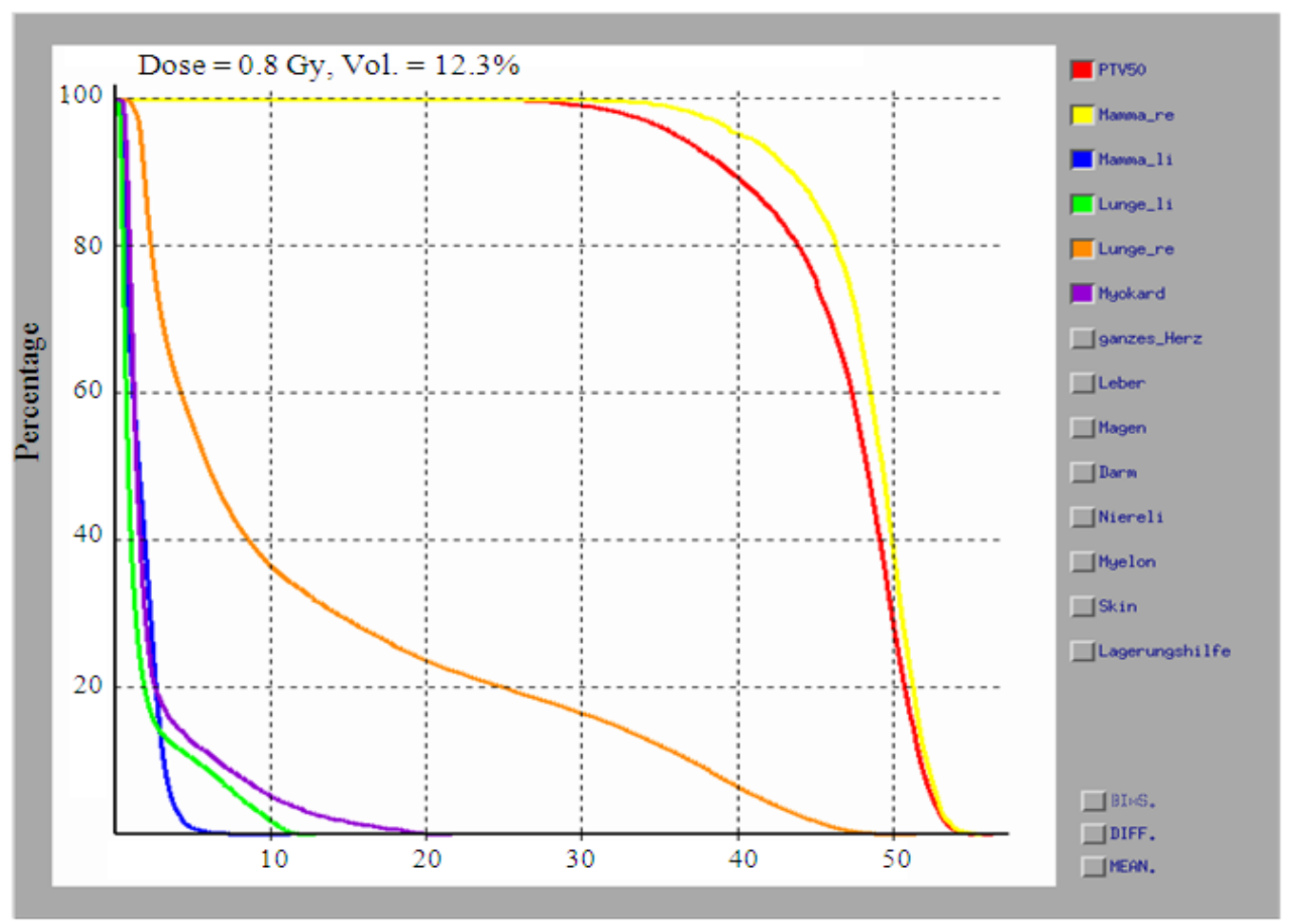

Figure. 4: DVH of the second plan

One can see from Figure 4 above that:

[. The target coverage was within the accepted values. Approximately, $90 \%$ of the breast and $80 \%$ of the PTV received 45 Gy or above.

[ Less than $10 \%$ of the epsilateral lung received $40 \mathrm{~Gy}$. The volume of that portion that received 20Gy (V20) was less than 25\%. This implies that the dose received by the whole lung was much smaller.

[ The volume of the heart receiving $30 \mathrm{~Gy}$ (V30) or above was negligible.

\section{Treatment planning times}

Optimization of the fields was performed by Hyperion using the stated constraints and cost functions set for the first patient. Using a computer with two processors, the treatment planning procedure, including contouring of planning target volume and organs at risk and preparing the CLN files, followed by the optimization, for the first CT data set took more than four hours. This is basically because calculations were performed on trial-and-error bases, bearing in mind the radiobiological parameters without a detailed plan from the radiotherapist. On approval the same values were re-used for the first plan. For this plan the time required for the pencil beam was 17 min and that for Monte Carlo calculations was $18 \mathrm{~min}$.

The optimized DVH result calculated for the CT data set after significantly altering the constraints for the right lung and heart referred to as the second plan 
is shown in Figure 5 . The time taken for this plan was $8 \mathrm{~min}$ for pencil beam calculations followed by $9 \mathrm{~min}$ for Monte Carlo calculations. The system had to rearrange the beam segments and to perform two calculations.

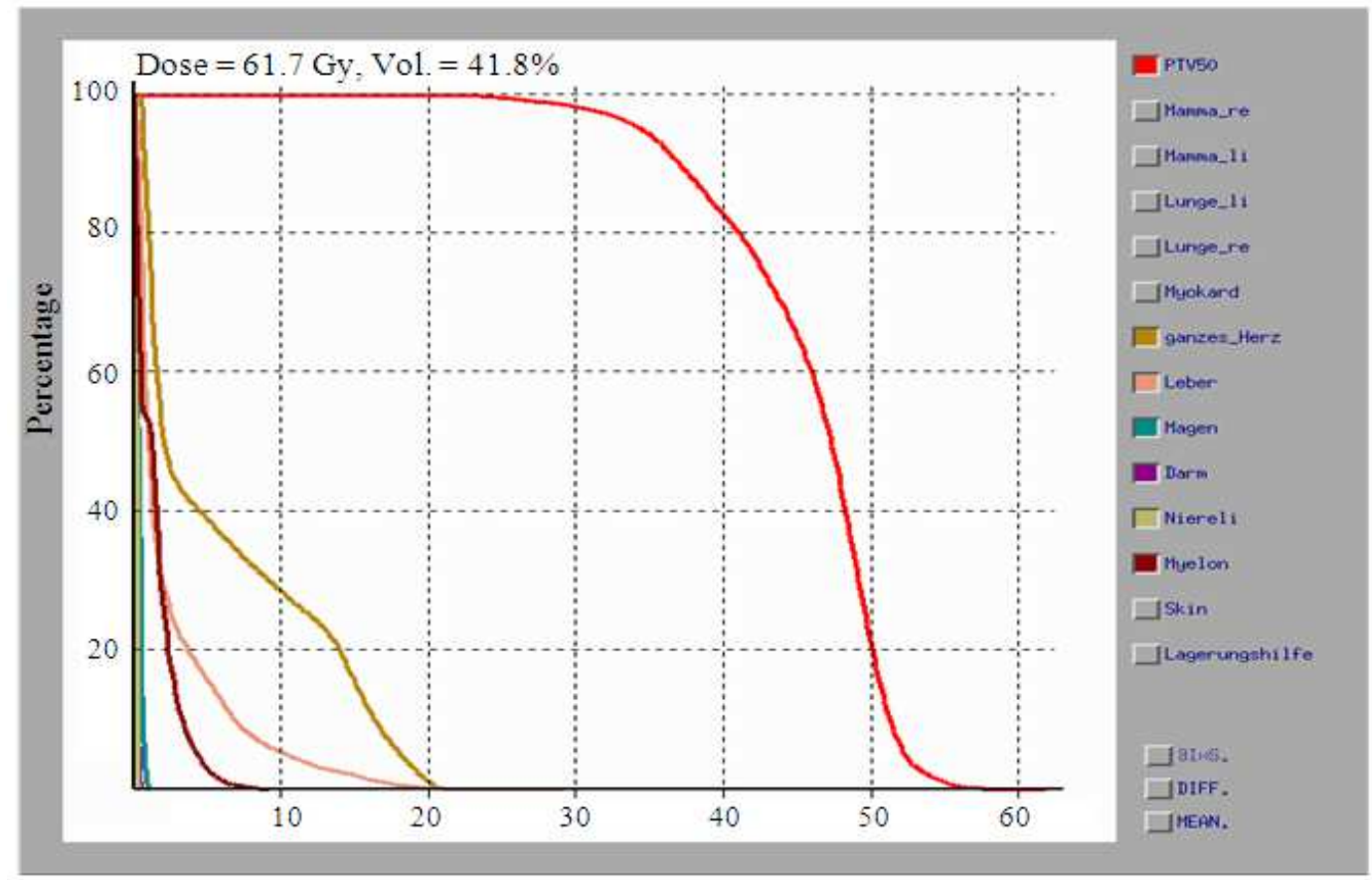

Figure 5: DVH of the third plan

There is a clear under-dosage of the PTV but the main interest in our study was to evaluate the time necessary to optimize the treatment rather than to evaluate the performance of Hyperion which is benchmarked and well documented.

The time taken after a slight alteration in the value of the PTV constraint, referred to as the fourth plan was only $2.5 \mathrm{~min}$. No re-segmentations or pencil beam calculations were necessary in this case, which explains the short duration required to optimize the plan. The time needed for optimized treatment planning can be significantly reduced with faster computers or computers with multiple processors using Hyperion since this code allows parallel processing.

\section{Discussion}

The radio-therapeutic doses received by the patient are limited by the tolerance of the normal tissues. Radiation is an unusual toxic agent, because the time of expression of cellular injury can be very variable from one tissue and tumour to another. Moreover, different patients given a standardized treatment can exhibit a range of normal tissue reactions. Thus, there is both dose dependence and variability 
in individual radio-sensitivity [10]. The lung and the heart are important dose limiting organs for radiation therapy of tumours in the thoracic region. The risks of pulmonary and cardiac toxicities depend on many factors amongst which are the volumes of organ irradiated by a certain threshold dose. Published data recording the percentage volume of the heart irradiated to various doses are limited. Such information might, however, be important in assessing radiation-related heart disease, because the percentage volume of the heart irradiated to a certain "threshold" dose might be a better predictor for cardiac death than, for instance, the mean heart dose or BED. Information is also needed concerning the effect of irradiating different cardiac structures, especially the coronary arteries. Coronary artery doses have been reported for several techniques, but only for left-sided irradiation; however, most studies have reported only the mean dose to the whole heart [3]. In recent studies evaluating the dose-volume effect for the lung and heart, a contributing risk factor for the development of pneumonitis was found to be the volume of the lung irradiated [5, 4]. Graham et al. [9] showed a lung V20 (volume that received $20 \mathrm{~Gy}$ ) to be an independent and significant predictor of GRADE2 or greater pneumonitis. The authors suggested that if $\mathrm{V} 20<25 \%$ the risk of pneumonitis is low. Moreover, V30 was observed by Gagliardi et al. [8] to be the threshold for calculated risks of ischemic heart diseases. V45 was associated with pathologic data that suggest the development of coronary vessels stenosis [11].

The inclusion of inhomogeneity corrections is crucial to the quality of treatment planning and consequently to the doses delivered to patients undergoing radiation therapy especially in regions of high heterogeneity like the thorax or when IMRT is needed. This is due to the combinatorial effects of many fields and the presence of steep fluence gradients [2].

Monte Carlo techniques are known to be the most accurate techniques for simulation of radiation transport in clinical situations and are expected to reproduce the measured absorbed dose to within $\pm 2 \%$ or $\pm 2 \mathrm{~mm}$ accuracy level, which are the requirements when evaluating treatment planning systems according to ICRU Report 42. Therefore they were used in the iterative optimization routine in Hyperion to overcome the complexities caused by inhomogeneities [13].

The motion of the critical organs is dealt with using contours of several CTs to calculate the virtual organ's Hull volume. This consists of the unity of deep inhale and exhale volumes plus an additional margin of $5 \mathrm{~mm}$. The deformable registration and other real-time deformable registration algorithms promise further solutions to the challenges of tumour motion. This will be implemented in many treatment planning systems in the near future and will allow higher tumour control probability without increasing the side-effects in healthy tissues [14].

Having accepted a protocol for treatment, the same .CLN file can re-used for any other data sets or patients with minor changes in some conditions so the time 
needed for each patient can be within the acceptable limits for routine work in a busy radiotherapy department.

\section{Conclusion}

Radiotherapy given after mastectomy (PMRT) will have a positive impact on the reduction of the risks of local recurrence. Research efforts have been devoted towards individualizing the radiation therapy process to each patient's specific anatomy and physiology in planning and delivery of high dose radiotherapy to avoid the aforementioned complications. These efforts have been encouraged by studies that indicate that higher doses can be safely delivered to limited healthy organs as well as the fact that higher target doses relate to improved tumour control.

Hyperion, the Monte Carlo based treatment planning system is easy to use and can calculate dose distributions in patients accurately. Challenges in PMRT such as tissue inhomogeneities, tumour motion and long times required for treatment planning can be effectively handled by Hyperion. Other Monte Carlo based treatment planning systems are also available and were also proved to be efficient. There is a need to validate these treatment planning systems for the different modalities such as electron, photons or combinations to allow the planner choose the optimum one for treatment. Radiotherapists and physicists should be trained to make use of such new techniques which will allow higher tumour control probabilities with minimum normal tissue complications.

\section{Acknowledgement}

Thanks to the staff at the University of Tuebingen: Markus Alber for facilitating for me to work with Hyperion, B. Frey for assistance in planning the presented patient cases and Matthias Soehn for his valuable remarks.

\section{References}

[1] Alber, M. and F. Nusslin, 1999. An objective function for radiation treatment optimization based on local biological measures. Phys Med Biol, 44:479-493

[2] Alber, M., M. Birkner, W. Laub and F. Nüsslin, 2000. Hyperion: An integrated IMRT planning tool. Proceedings of the XIII Conference on the use of Computers in Radiationtherapy. Hrsg. W. Schlegel, T. Bortfeld. Springer, Heidelberg, pp:46-48 
[3] Carolyn, W., T.A. Nisbet, P. Mcgale and S.C. Darby, 2007. Cardiac exposures in breast cancer radiotherapy: 1950s-1990s. Int. J Radiation Oncology Biol Phys, 69:1484-1495

[4] Clenton, S.J.. P.M. Fisher, J. Conway, P. Kirkbride and M.K. Hatton, 2005. The use of lung dose-volume histograms in predicting post-radiation Pneumonitis after non-conventionally fractionated radiotherapy for Thoracic Carcinoma. Clinical Oncology, 17:599-603

[5] Early Breast Cancer Trialists' Collaborative Group (EBCTCG), 2005. Effects of radiotherapy and of differences in the extent of surgery for early breast cancer on local recurrence and 15-year survival: an overview of the randomized trials LANCET, 366:2087-2106

[6] Fippel, M., M. Alber, M. Birkner, W. Laub and F. Nüsslin et al., 2001. Inverse treatment planning for radiation therapy based on fast Monte Carlo Dose calculation 2000 From: Advanced Monte Carlo for radiation physics, particle transport simulation and applications. Proceedings of the Monte Carlo 2000 Conference, Lisbon. Springer Berlin

[7] Fogliata, A.A., G.G. Nicolini, M.M. Alber, M.M. Asell and B.B. Dobler et al., 2005. IMRT for breast. Planning Study Radiother Oncol, 76:300-10. PMID 16153730

[8] Gagliardi, G., I. Lax, S. Soderstrom, G. Gyenes and LE. Rutqvist, 1998. Prediction of excess risk of long-term cardiac mortality after radiotherapy of stage I breast cancer. Radiother Oncol, 46:63-71

[9] Graham, M.V., J.A. Purdy, B. Emami, W. Harms and W. Bosch et al., 1999. Clinical dose-volume histogram analysis for pneumonitis after 3D treatment for Non-Small Cell Lung Cancer (NSCLC). Int. J. Radiation Oncology Biol Phys, 45:323-329

[10] Johansson, S., H. Svensson and J. Denekamp, 2002. Dose response and latency for radiation-induced fibrosis, edema and neuropathy in Breast Cancer patients. Int. J. Radiation Oncology Biol Phys, 52:1207-1219

[11] Krueger, E.A., M.J. Schipper, T. Koelling, R.B. Marsh and J.B. Butler et al., 2004. Cardiac chamber and coronary artery doses associated with postmastectomy radiotherapy techniques to the chest wall and regional nodes. Int. J. Radiation Oncology Biol Phys, 60:1195-1203

[12] Laub, W., M. Alber, M. Birkner and F. Nusslin, 2000. Monte Carlo dose computation for IMRT optimization. Phys Med Biol, 45:1741-1754 
[13] Papanikolaou, N., J.J. Battista, A.L. Boyer, C. Kappas and E. Klein et al., 2004. AAPM Report 85: Tissue inhomogeneity corrections for megavoltage photon beams Report of Task Group No. 65 of the Radiation Therapy Committee of the American Association of Physicists in Medicine 2004

[14] Sohn, M., M. Brinker, Y. Chi, J. Wand and D. Yan et al., 2008. Modelindependent, multimodality deformable image registration by local matching of anatomical features and optimization of elastic energy. Med Phys, 35:866-878 